\title{
Analysis on Several Typical Faults of Medical Linear Accelerator
}

\author{
Yun Sui \\ The second People's Hospital of Jinan City \\ China
}

\author{
Liang Guo, Shengui Zhang* \\ The Fouth People's Hospital of Jinan City \\ Taishan Medical College \\ China \\ *Corresponding author
}

\begin{abstract}
For many advantages, the medical linear accelerator has become an indispensable part in modern medical devices. However, due to the enormous system and a number of parts involved, the requirements to fault analysis and maintenance of medical accelerator is much higher. This paper introduces fault reasons, fault analysis and maintenance plan to several typical common faults of medical linear accelerator in detail, which is FLOW interlocking fault, MLC, dose rate instability fault, multi-leaf collimator fault and pressure-oil tank fault, which is significant to the maintenance work of medical linear accelerator to some extent.
\end{abstract}

Keywords- linearaccelerator; fault phenomenon; fault cause and fault maintenance

\section{INTRODUCTION}

With the development of medicine science and technology, the linear accelerator with traits of high accuracy, no damage to normal tis sue, short irradiation time and a good curative effect in the shallow and deep tumor is widely used in the clinic, which has become an important part of radiological devices today. Medical electron linear accelerator has become the most commonly used radiotherapy devices. Medical electron linear accelerator is used for the tumor radiation therapy. The electrons of medical electron linear accelerator get the energy from microwave electromagnetic accelerating field and accelerate along the straight orbit. Medical electron linear accelerator can be divided into traveling wave linear accelerator and standing wave linear accelerator depending on the form of accelerated electromagnetic field. The medical linear accelerator is mainly consist of accelerating system, electronic injection system, microwave generation and transmission system, pulse modulation system, vacuum system, constant-temperature cooling system, beam extraction system and power control system[1]. Once such a huge complicated system fails, it can be difficult to maintain if be not carefully studied its maintenance methods and steps. This paper introduces several typical faults of the medical linear accelerator and elaborates the fault reason and fault maintenance, which has certain reference significance for breakdown maintenance of the medical linear accelerator.

\section{FLOW INTERLOCKING FAULT}

\section{A. Fault Phenomenon}

The phenomenon of FLOW interlocking is common in medical linear accelerator. The example of fault: when FLOW interlocking phenomenon first appears, vacuum electric current of the main pump reaches 750 microamps and even is more than 950 microamps. The vacuumelectric current of electronic gun is 0 . After six months, when FLOW interlocking appears again, the linear accelerator cannot work.

\section{B. Fault Cause}

Usually, the causes of FLOW interlocking can be divided into the following several categories: the shortage of the cooling circulating water; excessive solid magnet; oil level is less than lower limit in the klystron. If FLOW interlocking phenomenon appears, check the pressure gauge of circulating water in the machine first to exclude the pump fault, pull the plug $\mathrm{J} 7$ in the klystron tank and check whether or not $\mathrm{C}$ and $\mathrm{D}$ pins access with a multimeter to exclude the oil level switch in the klystron tank is normal or not. Then measure the water flow switch with a multimeter. If it is found that the water flow s witch of accelerating tube is short circuit, it could be ascertained that whether the cause is the low flow rate of cooling water of the accelerating tube or flow sensor fault.

\section{Fault Analysis and Processing}

In terms of the fault, after ruling out the sensor fault, the most likely cause of cascading failure is the shortage of cooling water of the accelerating tube. At this time, feel the temperature of accelerating tube pipe is by hand touching, which is high. Test by a thermometer. It shows 37 degrees Celsius while the temperature of outlet pipe is 41 degrees Cels ius. Therefore, it can be tentatively concluded that the cooling water pipe in the accelerating tube may be blocked. Furthermore, discharge the cooling water in the accelerator first, dis connect the cooling flow sensor of accelerating tube, access the pipeline to airtight container and plug water input of flow sensor. Then start the pump switch, calculate outflow of water, disconnect cooling water inlet of accelerating tube and connect the water inlet. In this way, judge whether the cooling pipe has some congestion to lead to decreased water and causes the FLOW interlocking. 
Open inlet and outlet of the water pipe and find tiny particles stick to pipeline inwall, which may affect the water flow after scaling. The cause of blockage: the quality of circulating water is bad that leads to pipeline inwall scaling or attachments jamming in pipeline in wall, which causes the partial blockage and small water flow [2]. The way to deal with it is descaling and cleaning with scaling cleaner of air conditioning. First, prepare detergent and cleaning utensils, heat the distilled water and pour into detergent to dis solve dirt for about 5 minutes. Cover the entrance at the bottom with strainer to prevent suction of dross, access the outlet pipe to the plastic drum, rinse repeatedly the dissolving scale until the water is turbid and flowing with small pieces of material. Then continue to rinse for half an hour until the water is clear and has no color. Rinse repeatedly after dis solving scale; after the water turbidity, contain small pieces of material flow. Continue to rinse for half an hour until water is colorless.

\section{FAULT OF MULTI LEAFY COLLIMATOR}

\section{A. Fault Phenomenon}

Mechanical fault occurs most for multi leaf collimator. It mainly refers to the collimator blade cannot be in place in time, or does not reach the designated position, leaf blade spacing doesn't work (blade cannot stop in time and be "stuck") and backup blade can't accurately follow. Fault example: system error, MLC not ready (multi leaf collimator leaves are not ready).

\section{B. Fault Cause}

Such fault cause is relatively simple. It is mainly related to deformation of multi leafy collimator leaves, potentiometer of leaf blade, Limit switch malfunction, motor fault and the unreas onable radiation therapy prescription.

\section{Fault Analysis and Processing}

First, enter the interface of MLC service maintenance, check whether there is contact between multi leaf collimator leaves or "stuck" in the limit position of the leaves If there is, close the power supply of the multi leaf collimator and put back the leaves with special tools. If the same problem repeats in the same treatment, suggest the doctor modify the radiation treatment prescription. If there isn't, enter the part of service maintenance, choose diagnostic procedures of MLC (multi leaf collimator) and check the movement of every leaves [3]. When finding the motion of a leaf is ineffective, choose the high speed way and try to move it carefully. If it can be moved, it can continue to be used. After completion of treatment, apply a small amount of lubricating oil for radiation protection to lead screw of leaves. Pay attention to not apply with ordinary viscous oil to avoid increasing the resistance of the leaves since it will become solid paste after X ray irradiation. If it cannot be moved, check whether motor is damaged.

\section{THE FAULT OF DOSE RATE INST ABILITY}

\section{A. Fault Phenomenon}

It appears that 4,6, 9, 12,16,20MEV electronic line and 10MV photonic wire are normal and only dose rate of $6 \mathrm{MV}$ photonic wire is unstable.

\section{B. Fault Cause}

The fault of dose rate instability is common and there are many causes. The most common causes are the following two aspects. Firstly, the dose rate mainly changes with the output power of klystron. The output power decides the dose rate; if the oscillator frequency of klystron is unstable, the microwave power of accelerating tube is very unstable. Thus microwave can't be resonant with electron resonance well and electrons cannot be accelerated as required, which leads to dose rate instability. Secondly, the resonance of microwave and electronic is conducted in the accelerating tube. If the working state of the accelerating tube is suddenly changed, it will also change the dose rate, which is always ignored in the previous problem analysis. Temperature change and power grid voltage fluctuations both can influence the oscillator frequency of klystron.

\section{Fault Analysis and Processing}

If it is certain energy, check the change of the energy of the beamparameters at first. If there is any change, adjust according to the variation of dose rate. Firstly, shift switch AFc to manual shift gear and manually adjust the little red button AFC. Find dose rate changes, but it is unstable. Adjust the repetition frequency of H publication and 5011 electric current of speed line circle. But no matter how to adjust, the dose rate is still unstable. Eliminate the temperature influence on dose rate because other dose rate of energy beamis normal. Varian $2300 \mathrm{D}$ accelerators in the low energy mode (line $6 \mathrm{mv}$ photons) use energy switch to adjust electric field intensity in the back of accelerating tube [4]. The changes of energy switch greatly changed the energy spectrum and energy gap of electron bunching. The energy spectrumand energy gap directly impact on dose rate in and after electronic target. After opening the shell of machine GANTRY, check the power switch and find some loose in the power switch. After fixing the power switch tightly, open the machine and make it work. The dose rate of $6 \mathrm{Mv}$ photonic wire goes back normal.

\section{MULTi-LEAF COLLIMATOR FAULT}

\section{A. Fault Phenomenon}

After varian 23EX linear accelerator startup, the self-checking of accelerator MLC cannot pass.

\section{B. Fault Cause}

Check and find that the indicator light of MLC POWER DISTRIBUTION is darker than DS1,2,3.Then check the contact of DS4 indicator light and find it's only about $+2.6 \mathrm{~V}$ voltage that the normal power supply shall be $+5 \mathrm{~V}$. After restarting the MLC switch power supply, sometimes DS4 power supply is normal 
and sometimes not. When DS4 indicator light is normal, the voltage is $+5 \mathrm{~V}$. When DS4 indicator light is abnormal, the voltage is $+2.6 \mathrm{~V}$. Since the MLC power supply supplies for MLC POWER DISTRIBUTION board first and then MLC POWER DISTRIBUTION board will supply and load electricity through the J1, J2, J4 respectively. J1 supplies side A, J2 supplies side B and J4 supplies MISCPWR. However, when pulled out one of J1 and $\mathrm{J} 2$, DS4 indicator light is normal. When $\mathrm{J} 1$ and $\mathrm{J} 2$ both exist, DS4 could dis play abnormal. So the cause may be the power load is too large, so the power protection circuit works to stop its operation.

\section{Fault Analysis and Processing}

Since the cause of this phenomenon is the heavily power load, so analysis this section of the circuit, that is integrated circuit plate of MLC POWER DISTRIBUTION. This section of the circuit is $+5 \mathrm{~V}(\mathrm{PS}) \rightarrow \mathrm{J} 3: 16(15) \rightarrow \mathrm{F} 1(10 \mathrm{~A}) \rightarrow \mathrm{FL} 1$ (inductance) which goes 8 feet out, back to 2 feet and then output by 7 feet. The inductance is buffering and eliminating in the switch of power and supply $+5 \mathrm{~V}$.After 7 feet output, there is a power signal returning to the $\mathrm{J} 3: 14(+5 \mathrm{VSENSE}+$ ). This $+5 \mathrm{~V}$ SENSE+ will control the output voltage of J3:16. If the voltage is reduced when it returns to the switch power, then the output of J3:16 will be lower. This is a feedback circuit, so J3:16 is turning to $+2.6 \mathrm{~V}$ [5].

Disconnect the feedback signal of FL1-7 +5V SENSE+ first, so the signal of FL1-1 after J3:16 will not be $+2.6 \mathrm{~V}$. In this way, the feedback to J3:16 and total voltage of TP5 will be the normal $+5 \mathrm{~V}$. The $+5 \mathrm{~V}$ voltage and the voltage of the voltage circuit will all be $+5 \mathrm{~V}$. By this method, the TP5 voltage of MLC POWER DISTRIBUTION board will go back to $+5 \mathrm{~V}$ and DS4 indicator light also returns to normal brightness. Self-ins pection is over.

\section{PRESSURE-OIL T ANK FAULT}

\section{A. Fault Phenomenon}

The machine reports 30th interlocking "MODULATORINCOMPLETE" and the light of 30th interlocking "CHARGESYSTEM" is off, so the charging system fails. Observe the light "IS" is off and doubt there are problems in thyratron or high pressure tank. Observe there is water on the ground and in the accelerator klystron. Open the shield of klystron and find there is rupture in the inner loop water pipe of the cooling klystron. Observe the inner loop water tank and find circulating water leakage, which is about 10 13 L. Observe the oil level of the oil tank is turbid, which should be transparent and clearly seen.

\section{B. Fault Cause}

"IS" means secondary modulator current. "IS" will produce interlock if it is detected existence and stronger than a certain level. "IS" interlocking is incurred owing to: the insulation of transformer oil decline, charging diode damage, thyratron malfunction, reverses diode, etc. Considering that the machine breaks down in the absence of people, and the machine has not been in high pressure condition. Therefore, the failure should be generated when the machine is in this condition by the water, which is from the internal circulating pipe burst in the cooling klystron, and it infiltrates into the fuel tank. Under this condition, with the water recycling and oil circulating proceeding normally, the machine does not raise the alarm until the internal cycling water level hits the bottom and the permeating internal cycling water and oil mix together by oil circulating, which gives rise to a decline of oil insulation. Since the voltages of the thyratron are detected normal, the high-pressured oil tank is the "culprit" of "IS" failure.

\section{Fault Analysis and Processing}

Due to the fact that the machine is not in high-pressure state, analysis tells that the thermistors in the high-pressure tank, charging diode, reverse diode and pulse transformer are not worn. The machine should be in running order if the oil is drained and new oil is used, therefore it is concluded that the "IS" failure is caused by the high pressure tank. Firstly, discharge the internal and external circulating water, dis mantle the cooling pipe and output conduit of the klystron, as well as the wiring linking the klystron and fuel tank, then dismantle the hydrator after removing with the special tools the klystron and cock coil.

Secondly. Turn off the power on each part of the machine, except for the oil pump circuit power. Draw the oil in the fuel tank with the original oil pump to an oil bucket. In the pumping process it is noticed that the oil is milky white and foamy, indicating that oil and water are mixed and can no longer be used. Because of residual oil in the tank, the great deal of white oil-bearing foamon the pulse transformer and thyristors must be further processed. First extract the remaining oil with a small pump, and then repeatedly rinse the oil tank with a $50 \mathrm{~kg}$ transformer oil-use pump and pump the water out until no oil foamand impurities exist in the tank. The whole washing process comes to an end after the oil tank is washed with some clean trans former oil, and water is extracted. After this, bake the oil tank with a infrared lamp to make all parts dry. Reinstall all the parts and wiring as it was before and do the working adjustment [6]. The first step is to enter the engineer mode, reducing the PFN parameters so as to check the normality of thyristor voltage. Observe the: "IP" and "IS" waveform with an oscilloscope, the waveform is normal.

\section{SUMMARY}

With high failure rate, complex structure and advanced technology, the medical linear accelerator, collecting microwave, gas, electricity and water, is advanced high-tech equipment. It is necessary to strengthen the daily maintenance and protection work of the medical linear accelerator to keep it in good working condition. A swift handling of the running failures will boost work efficiency and ensure that a good service can be provided to the clinical treatment of patients.

\section{REFERENCES}

[1] Ruiyao Jiang. Analyze and summarize the troubles of linear accelerator for 10 years, J. Medical Equipment.2011, 18(11): 36-38. 
[2] Qi Ke, Shaofu Huang, Bin Huang. Analysis and maintenance for FLOW malfunction of varian 23EX linear accelerator, J. Guide of China Medicine.2013,11(20):786-787.

[3] Quanhai Ma, Xiaojuan Zhai. Elekta Linear Accelerator Multi-leaf Collimator (MLC) Failure Analysis and Maintenance, J. Operation and Guarantee for Instrument.2011, 26(7): 113,132 .

[4] Lingguang Meng, Hongchang Lei, Leijie Ma. Disposal and maintenance of unstable dose rate of the VARIAN CL 2300C/D medical linear accelerator, J. Medical Equipment. 2009, 22(8): 115-117.

[5] Wei Hua, Jun Li. Troubleshooting to MLC of varian 23EX linear acceleraor, J. Operation and Guarantee for Instrument.2011, 26(1): 100-101.

[6] Jun Chen, Bingjian Du. Siemens linear accelerator fault repair one case of high-pressure tank, J. Chinese Medical Equipment Journal.2008,29(6):116-117. 